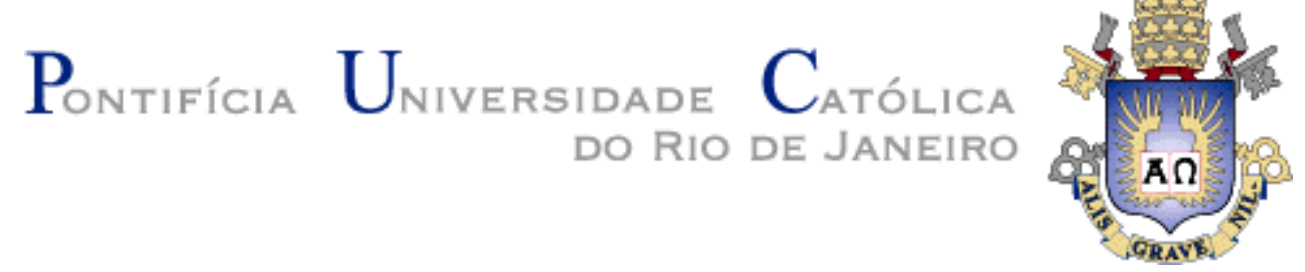

Heron Vilela de Oliveira e Silva Autoria Hipermídia DissertaÇão de MESTRADO

DEPARTAMENTO DE INFORMÁTICA

Programa de Pós-Graduação em Informática 


\section{Pontifícia $U_{\text {niversidade }}$ Católica $_{\text {a }}$ \\ DO RIO DE JANEIRO \\ Heron Vilela de Oliveira e Silva}

\section{X-SMIL: Aumentando Reuso e Expressividade em Linguagens de Autoria Hipermídia}

Dissertação de Mestrado

Dissertação apresentada como requisito parcial para obtenção do título de Mestre pelo Programa de PósGraduação em Informática da PUC-Rio.

Orientadore: Luiz Fernando Gomes Soares Co-orientador: Rogério Ferreira Rodrigues 
Heron Vilela de Oliveira e Silva

\title{
X-SMIL: Aumentando Reuso e Expressividade em Linguagens de Autoria Hipermídia
}

\begin{abstract}
Dissertação apresentada como requisito parcial para obtenção do título de Mestre pelo Programa de PósGraduação em Informática da PUC-Rio. Aprovada pela Comissão Examinadora abaixo assinada.
\end{abstract}

Luiz Fernando Gomes Soares Orientador

Departamento de Informática - PUC-Rio

Rogério Ferreira Rodrigues Co-orientador

Departamento de Informática - PUC-Rio

Marco Antonio Casanova

Departamento de Informática - PUC-Rio

Renato Fontoura de Gusmão Cerqueira

Departamento de Informática - PUC-Rio

José Eugenio Leal

Coordenador Setorial do Centro Técnico Científico - PUC-Rio 
Todos os direitos reservados. É proibida a reprodução total ou parcial do trabalho sem autorização da universidade, do autor e do orientador.

\section{Heron Vilela de Oliveira e Silva}

Formou-se em Engenheira de Computação pela PUC-Rio em 2002. Atualmente, integra o grupo de pesquisadores do Laboratório TeleMídia da PUC-Rio, desenvolvendo pesquisa na área de Sistemas Hipermídia.

Ficha Catalográfica

Silva, Heron Vilela de Oliveira e

X-SMIL: aumentando reuso e expressividade em linguagens de autoria hipermídia / Heron Vilela de Oliveira e Silva ; orientador: Luiz Fernando Gomes Soares. - Rio de Janeiro : PUC-Rio, Departamento de Informática, 2005.

210 f. : il. ; $30 \mathrm{~cm}$

Dissertação (mestrado) - Pontifícia Universidade Católica do Rio de Janeiro, Departamento de Informática .

Inclui referências bibliográficas

1. Informática - Teses. 2. Sistemas hipermídia. 3. Autoria. 4. Linguagens declarativas. 5 Relações. 6. SMIL. 7. Sincronização. 8. Conectores. 9. Templates. 10. NCL. I. Soares, Luiz Fernando Gomes. II. Pontifícia Universidade Católica do Rio de Janeiro. Departamento de Informática. III. Título. 


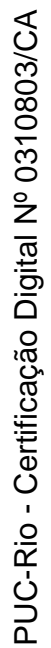

Este trabalho é dedicado:

À Deus, por ser. 


\section{Agradecimentos}

À minha família, pelo amor incondicional.

Aos meus orientadores, pela amizade, compreensão e apoio constante.

Aos amigos, por estarem, mesmo quando ausentes, sempre presentes.

Ao espírito TeleMídia.

Ao DI.

À PUC, CAPES, CNPq e FUNTTEL. 


\section{Resumo}

Silva, Heron Vilela de Oliveira. X-SMIL: Aumentando Reuso e Expressividade em Linguagens de Autoria Hipermídia. Rio de Janeiro, 2005. xxxp. Dissertação de Mestrado - Departamento de Informática, Pontifícia Universidade Católica do Rio de Janeiro.

Este trabalho está inserido no contexto de ambientes de autoria e execução hipermídia, sendo as linguagens declarativas para autoria de documentos o seu foco principal. Tendo-se como objetivo aumentar a expressividade e o reuso na especificação de documentos hipermídia, este trabalho introduz as linguagens XSMIL e NCL - Nested Context Language - versão 2.1. Utilizando-se o conceito de templates, X-SMIL permite a definição de novas semânticas para composições SMIL, além dos tradicionais elementos seq, par e excl. Templates, em X-SMIL, são especificados em um perfil de XTemplate, que estende a idéia original da linguagem XTemplate de NCL. Com base nas novas facilidades para definição de templates, esse perfil foi usado para especificar a linguagem NCL 2.1. X-SMIL também permite a especificação de conectores hipermídia, tratando relações hipermídia como entidades de primeira classe - funcionalidade incorporada em XSMIL pelo uso do módulo XConnector de NCL. Outro objetivo deste trabalho é o de apresentar um framework para o processamento de documentos XML. Utilizando-se esse framework, diversos compiladores foram implementados, o que possibilitou, entre outras funcionalidades, a conversão de documentos NCL em especificações SMIL ou X-SMIL e vice-versa.

\section{Palavras-chave}

sistemas hipermídia; autoria; linguagens declarativas; relações; elos; sincronização; conectores; templates; NCL; SMIL; XML; compilador 


\section{Abstract}

Silva, Heron Vilela de Oliveira. X-SMIL: Improving Reuse and Expressiveness in Hypermedia Authoring Languages. Rio de Janeiro, 2005. XXXp. Master Thesis - Departamento de Informática, Pontifícia Universidade Católica do Rio de Janeiro.

This work is related to hypermedia authoring and execution environments, and its main focus is declarative document authoring. Aiming at improving the expressiveness and reuse in the specification of hypermedia documents, this work introduces the hypermedia authoring languages X-SMIL and NCL - Nested Context Language - version 2.1. Exploiting the concept of templates, X-SMIL allows the definition of new semantics for SMIL compositions, besides its usual seq, par and excl elements. X-SMIL templates are specified using an XTemplate profile, which extends the original idea of the NCL XTemplate language. Bringing new facilities for template definitions, this new profile is used to further improve the NCL language. X-SMIL also offers support for handling hypermedia relations as first-class entities, through the use of hypermedia connectors - brought to X-SMIL via the NCL XConnector module. Another important goal of this work is to present a framework to facilitate the development of XML documents parsing and processing tools. Based on this framework, several compilers were implemented, permitting, among other features, the conversion of NCL documents into SMIL or X-SMIL specifications and vice-versa.

\section{Palavras-chave}

hypermedia systems; authoring; declarative languages; relations; links; synchronization; connectors; templates; NCL; SMIL; XML; compiler 


\section{Sumário}

1 Introdução 15

1.1. Motivação 15

$\begin{array}{ll}\text { 1.2. Objetivos } & 19\end{array}$

1.3. Organização da Dissertação 22

2 Linguagens para Descrição de Documentos Hipermídia 23

2.1. SGML e XML $\quad 24$

2.2. HTML e XHTML 26

2.3. SMIL 27

$\begin{array}{ll}\text { 2.4. NCL } & 29\end{array}$

3 Nested Context Language 2.1 34

3.1. Funções de Custo 34

3.2. Regras de Apresentação 36

3.3. Refinamentos de NCL $2.1 \quad 40$

3.4. Linguagem XConnector 46

3.5. Linguagem XTemplate $\quad 51$

4 X-SMIL $\quad 64$

4.1. XT-SMIL: SMIL + XTemplate 64

4.2. SMIL + XConnector (XC-SMIL) e X-SMIL 70

5 Framework para Compiladores $\quad 74$

5.1. Framework Genérico para Processamento 76

5.2. Framework para Compiladores de Documentos NCL 85

5.2.1. Funcionamento do Framework de Compiladores NCL 86

5.2.2. Compiladores de documentos NCL 89

5.3. Framework para Compiladores de Documentos SMIL 93

5.4. X-SMIL 95 
6 Trabalhos Relacionados 107

6.1. Template de Composição 107

6.2. Processamento de documentos XML 110

6.3. Framework e Compiladores 112

6.4. Compiladores XML 117

6.5. Extensões à SMIL 118

6.6. Conversão entre Modelos 120

7 Conclusão e Trabalhos Futuros 123

7.1. Templates 124

7.2. Conversões entre Formatos 127

8 Referências Bibliográficas 130

9 Apêndice A 136

9.1. NCL 2.0

9.2. NCL 2.1

10 Apêndice B 142

10.1. NCL21.xsd 142

10.2. NCL-Attributelnterface.xsd 144

10.3. NCL-BasicComposite.xsd 144

10.4. NCL-BasicDescriptor.xsd 145

10.5. NCL-BasicLayout.xsd 145

10.6. NCL-BasicMedia.xsd 146

10.7. NCL-BasicTiming.xsd 147

10.8. NCL-component.xsd 147

10.9. NCL-CompositeConnector.xsd 148

10.10. NCL-CompositeDescriptor.xsd 149

10.11. NCL-Compositelnterface.xsd 149

10.12. NCL-compositeTemplate.xsd 150

10.13. NCL-connector.xsd 150 
10.14. NCL-ContentControl.xsd

10.15. NCL-control.xsd 152

10.16. NCL-CostFunction.xsd 153

10.17. NCL-DescriptorControl.xsd 154

10.18. NCL-interface.xsd 154

10.19. NCL-Language.xsd 157

10.20. NCL-layout.xsd 172

10.21. NCL-link.xsd 174

10.22. NCL-Linking.xsd 175

10.23. NCL-Medialnterface.xsd 176

10.24. NCL-presentation.xsd 177

10.25. NCL-struct.xsd 178

10.26. NCL-Structure.xsd 179

10.27. NCL-SwitchInterface.xsd 179

10.28. NCL-TestRules.xsd 180

10.29. NCL-timing.xsd 180

10.30. NCL-XTemplateUse.xsd 182

11 Apêndice C 183

11.1. XConnector21.xsd 183

12 Apêndice D 195

12.1. XT-BasicContraints.xsd 195

12.2. XT-BasicLinking.xsd 195

12.3. XT-BasicResources.xsd 196

12.4. XT-BasicVocabulary.xsd 197

12.5. XT-connector.xsd 197

12.6. XT-ConnectorVocabulary.xsd 198

12.7. XT-constraints.xsd 198

12.8. XTemplate21.xsd 199

12.9. XT-language.xsd 200

12.10. XT-linking.xsd 206

12.11. XT-resources.xsd 207

12.12. XT-struct.Xsd 207 
12.13. XT-Structure.xsd

208

12.14. XT-vocabulary.xsd 


\section{Lista de figuras}

Figura 1:1. Subsistemas de um sistema hipermídia 16

Figura 2:1 - Aplicação SGML. 25

Figura 2:2 - Exemplo de um documento SMIL. 29

Figura 2:3 - Exemplo de um documento NCL. 30

Figura 2:4 - Exemplo de elos NCL e de reuso de conectores. 31

Figura 2:5 - Exemplo do uso de templates de composição. 33

Figura 3:1 - Exemplo de funções de custo em NCL 2.1. 35

Figura 3:2 - Exemplo de um nó switch em NCL 2.0

Figura 3:3 - Exemplo de regras de apresentação e nó switch em NCL 2.1.3

Figura 3:4 - Exemplo de documento NCL 2.1. 42

Figura 3:5 - Switch de conteúdo e de descritores. 44

Figura 3:6 - Máquina de estados de um evento. 47

Figura 3:7 - Exemplos de conectores em NCL 2.0. 49

Figura 3:8 - Relações finishes e overlaps. 49

Figura 3:9 - Exemplo de conectores em NCL 2.1. 50

Figura 3:10 - Exemplo de template de composição em NCL 2.0. 53

Figura 3:11 - Visão temporal de uma composição NCL. 53

Figura 3:12 - Exemplo de template de composição em NCL 2.1. $\quad 59$

Figura 3:13 - Visão temporal de uma composição NCL. 60

Figura 3:14 - Exemplo de template com relações de inclusão e por conectores em NCL 2.1. 61

Figura 4:1 - Visão temporal de uma composição par em SMIL. $\quad 66$

Figura 4:2 - Visão estrutural de uma composição XT-SMIL par antes e após o processamento de template. 66

Figura 4:3 - Template audioComLegendasEnPt em XT-SMIL. 68

Figura 4:4 - Composição XT-SMIL par utilizando um template. $\quad 70$

Figura 4:5 - Resultado do processamento de template em uma composição XT-SMIL par. $\quad 70$

Figura 4:6 - Exemplo de elo em uma composição SMIL. 
Figura 4:7 - Exemplo de elo em uma composição XC-SMIL.

Figura 4:8 - Exemplo de elos multiponto em uma composição XC-SMIL. 72

Figura 4:9 - Exemplo de uma composição X-SMIL.

Figura 5:1 - . Visão geral da estruturação em dois níveis dos frameworks para compiladores de linguagens modulares.

Figura 5:2 - Exemplo de um método do tipo parse de um framework de compiladores.

Figura 5:3 - Diagrama de classes do gerador automático de frameworks de compiladores.

Figura 5:4 - Diagrama de classes do framework para compiladores NCL.86 Figura 5:5 - Exemplo simplificado de documento NCL. 87

Figura 5:6 - Compiladores NCL. 90

Figura 5:7 - Composição SMIL gerada a partir do compilador NCL-SMIL.92 Figura 5:8 - Diagrama de classes do Framework para Compiladores SMIL.9 Figura 5:9 - Processador de Template XTemplate 2.1.

Figura 5:10 - Documento XML com a cópia de uma composição sendo processada.

Figura 5:11 - Composição gerada pelo processador de templates. $\quad 99$

Figura 5:12 - Exemplo de transformada body XSLT. 101

Figura 5:13 - Exemplo de transformada link XSLT. 104

Figura 5:14 - Exemplo de transformada constraint XSLT. 105

Figura 6:1 - Exemplo de um documento representando um noticiário no sistema LAMP.

Figura 6:2 - Template para artigos de um noticiário. 109

Figura 6:3 - API DOM de JAXP. 110

Figura 6:4 - API SAX de JAXP.

Figura 6:5 - Ferramentas XANTLR e TDOM. 114

Figura 6:6 - Especificação da classe FirstApplet em Java. 115

Figura 6:7 - Especificação de FirstApplet em JavaML. 116

Figura 6:8 - Framework XVM para o desenvolvimento de aplicações com XML.

Figura 6:9 - Edição de um documento em timeline. 


\section{Lista de tabelas}

Tabela 1 - Elementos do módulo CostFunctions.

Tabela 2 - Elementos do módulo TestRules. 38

Tabela 3 - Diferenças entre NCL 2.1 e NCL 2.0. 46

Tabela 4 - Nomes das transições para a máquina de estados de um evento. $\quad 47$

Tabela 5 - Elementos da linguagem XTemplate de NCL 2.0. 52

Tabela 6 - Elementos da linguagem XTemplate de NCL 2.1. 56

Tabela 7 - Tabela de Elementos.

Tabela 8 - Tabela de Grupos de Elementos. 77

Tabela 9 - Tabela de Áreas Funcionais. 